\title{
Segregação socioespacial e oferta de serviços de saúde na Região Metropolitana de Belo Horizonte em 2010*
}

\author{
Socio-spatial segregation and health services provision \\ in the Metropolitan Region of Belo Horizonte in 2010 \\ Lucas Wan Der Maas [l] \\ Erick de Oliveira Faria [II] \\ Júlia Leite de Carvalho Fernandes [III]
}

\section{Resumo}

0 objetivo deste artigo é analisar a relação entre segregação socioespacial e oferta de serviços de saúde na Região Metropolitana de Belo Horizonte em 2010. Foram utilizados dados do Censo Demográfico, do Cadastro Nacional de Estabelecimentos de Saúde e da Relação Anual de Informações Sociais. As informações foram organizadas em áreas de ponderação por meio da extração de coordenadas geográficas e analisadas com estatística descritiva e correlação espacial. Os resultados apontam que os serviços públicos de atenção primária à saúde concentravam-se em áreas onde predominavam grupos socioocupacionais inferiores na hierarquia social, ainda que a disponibilidade de médicos não acompanhasse a mesma lógica. Os serviços privados localizavam-se em áreas segregadas por grupos intermediários e superiores, situados principalmente na capital.

Palavras-chave: segregação socioespacial; serviços de saúde; análise espacial; Região Metropolitana de Belo Horizonte; correlação espacial.

\begin{abstract}
The aim of this article is to analyze the relationship between socio-spatial segregation and provision of health services in the Metropolitan Region of Belo Horizonte in 2010. Data from the Population Census, the National Register of Health Establishments and the Annual Record of Social Information were used. The data were organized in weighting areas through the extraction of geographic coordinates and analyzed through descriptive statistics and spatial correlation. The results show that the public primary care services were concentrated in areas where lower socio-occupational groups in the social hierarchy predominated, although the availability of doctors did not follow the same logic. Private services, in turn, were located in areas segregated by intermediate and higher groups, mainly in the capital city.
\end{abstract}

Keywords: socio-spatial segregation; health services; spatial analysis; Metropolitan Region of Belo Horizonte; spatial correlation. 


\section{Introdução}

0 presente artigo apresenta resultados de um estudo cujo objetivo era analisar a associação entre segregação socioespacial e oferta de serviços de saúde. Entendemos que ambos os fenômenos se conformam simultaneamente como estruturas e produtos dos processos mais amplos de hierarquização social que ocorrem no espaço urbano. Investigamos especificamente a ação do Estado e a do mercado enquanto agentes da segregação urbana através da análise da oferta e da distribuição de equipamentos de saúde do Sistema Único de Saúde (SUS) e do mercado privado (Saúde Suplementar), respectivamente, e da análise da relação dessa distribuição com a hierarquia social urbana. Para tanto, realizamos um estudo quantitativo a partir da conjugação de diversos dados secundários referentes à Região Metropolitana de Belo Horizonte (RMBH), em 2010, e da aplicação de técnicas estatísticas de correlação.

Este trabalho está dividido em quatro partes, além desta introdução e das considerações finais. Inicialmente, recuperamos alguns conceitos centrais para o estudo da produção do espaço em saúde. Na sequência, apresentamos a metodologia, detalhando a definição das categorias sociais, das unidades espaciais e dos procedimentos estatísticos utilizados. Nas duas seções seguintes, descrevemos e discutimos os resultados. Na primeira, apresentamos os dados referentes à distribuição da hierarquia social e da oferta de serviços de saúde e, na segunda, analisamos a associação entre as duas dimensões.

\section{Espaço e saúde}

Nas últimas décadas, desde a criação do Sistema Único de Saúde (SUS) pela Constituição de 1988, a questão do espaço tem ganhado importância no cenário das políticas públicas de saúde. Isso acontece, entre vários motivos, pela necessidade de consolidar e/ou qualificar os processos de regionalização e construção de redes de serviços de assistência à saúde de forma a diminuir as distâncias sociais existentes no acesso à saúde. Ocorre, portanto, pelo imperativo de aproximar os serviços de saúde do cidadão através da alocação de equipamentos e profissionais de saúde, sobretudo médicos, em regiões consideradas desassistidas em termos de insuficiência das ações públicas. Tais regiões são geralmente pobres, revestidas de graves problemas de saúde e com sérias barreiras de acesso, sejam elas físicas, sociais ou culturais. Não são poucos os esforços estatais já logrados ou em curso que objetivam dirimir as desigualdades de acesso aos serviços de saúde. Muitas experiências brasileiras, inclusive, foram e ainda são orientadas por estudos de identificação das desigualdades espaciais com o objetivo de alocar recursos físicos e humanos direcionados a áreas com maiores necessidades de saúde, como o caso do Programa Mais Médicos ${ }^{1}$ (Girardi et al., 2016).

A relevância do espaço para a saúde tem despertado interesse de pesquisadores de várias disciplinas que dialogam mais ou menos entre si, como sociologia urbana, saúde coletiva, epidemiologia, geografia da saúde e análise de políticas públicas. No Brasil, a frequência dos estudos sobre o tema aumentou 
consideravelmente nos últimos anos, não só em função do contexto político e do interesse dos pesquisadores mencionados, mas também pelo avanço das ferramentas de geoprocessamento, além da melhoria das informações e das estatísticas públicas disponíveis (Souza et al., 2016). Em sua maioria, porém, prevalecem estudos embasados nas concepções de fatores de risco e na vertente ecológica da doença, no campo da epidemiologia, os quais são principalmente descritivos e com pouca problematização sobre as ações sociais que conformam o espaço. Tais estudos são predominantes até hoje, mesmo após o chamado movimento da epidemiologia crítica ou social, dos anos 1970, que, entre outras ações, procurou superar a abordagem ecológica clássica, introduzindo a noção de espaço como construção social (Monken et al., 2008). Embora alguns trabalhos sejam herdeiros desse movimento, são ainda menos comuns os que buscam compreender as possíveis correlações entre desigualdades sociais, saúde e espaço. No campo das ciências sociais, tais estudos também são escassos, em que pese o profícuo campo dos estudos de sociologia urbana no Brasil.

Neste trabalho, optamos por trabalhar com o conceito de espaço ${ }^{2}$ a partir do ponto de vista das ciências sociais. Nesse sentido, para além da dimensão física, importa entendê-lo como lugar no qual as ações sociais ocorrem. É evidente que o espaço possui uma realidade física apriorística, mas a perspectiva adotada permite pensá-lo como o lugar em que as ações sociais dão sentido à vida no transcorrer das relações humanas. Nessa perspectiva, entende-se que: o espaço (1) molda a ação dos atores sociais; (2) orienta os significados atribuídos por esses atores; (3) é o cenário imediato de onde a ação se desenvolve; e (4) afeta as consequências da ação, ao passo que também é resultado dela (Therborn, 2004).

Do ponto de vista macrossociológico, é possível verificar empiricamente como o espaço está organizado, tendo em vista sua conformação histórica em torno de ações sociais que vão produzindo formas específicas de ocupação e apropriação. Na perspectiva proposta por Bourdieu (2003), o espaço físico pode ser apreendido como uma retradução do espaço social, sendo este caracterizado pelas posições relativas que os indivíduos preenchem. Assim, o espaço físico apresenta-se através da objetivação das distâncias sociais simbolicamente construídas no espaço social, isto é, da disposição dos agentes sociais, uns em relação aos outros. Ainda conforme o autor, como as distâncias sociais operam por meio de exclusão, distinção e hierarquização, o espaço físico tende a se estabelecer a partir da concentração de áreas opostas, sendo umas consideradas valorizadas e nobres, e outras, carentes e empobrecidas.

Os processos de divisão social que levam determinados grupos a se localizarem de forma concentrada, isto é, homogeneamente, em áreas geográficas específicas, em oposição a outros grupos e áreas, são chamados de segregação. Como destaca Moya (2011), o conceito de segregação é utilizado na teoria social para explicitar a separação de grupos ou classes sociais em diferentes áreas da cidade. Segundo a autora, de forma explícita ou não, todos os estudos têm convergido para essa noção geral, destacando o aspecto social na conformação dos espaços urbanos. Complementarmente, recorrendo à definição bourdiana de espaço, podemos definir segregação socioespacial como um conjunto de divisões no espaço 
físico derivado de desigualdades existentes na ocupação e na apropriação do espaço social de acordo com a posição social dos agentes.

Para introduzir a questão da saúde nessa concepção sobre espaço e suas divisões, é necessário compreender que a posição dos agentes sociais é multideterminada pelos diferentes capitais por eles apropriados nos campos da vida social (político, econômico, cultural, social) (Bourdieu, 2003), nos quais a saúde pode ser entendida como um recurso transversal. Isso significa percebê-la como um bem que perpassa todos esses campos e que confere, aos agentes sociais, ganhos de localização no espaço social. Esses ganhos são objetivados no espaço físico através do acesso a equipamentos de saúde, que, por sua vez, estão hierarquizados segundo valores econômicos e simbólicos distintos. Decorre dessa noção que os serviços de saúde mais caros e de maior prestígio - em geral, os de maior complexidade, associados a tecnologias e grandes equipamentos hospitalares - tenderão a se localizar em áreas apropriadas por agentes sociais mais capitalizados. Em outras palavras, as divisões sociais derivadas das ações relativas à saúde são produtos coletivos dos processos mais amplos de hierarquização social que historicamente ocorrem no espaço, ao mesmo tempo que produzem divisões segundo a apropriação que os agentes fazem dos bens de saúde disponíveis.

\section{Material e métodos}

Empiricamente, buscamos identificar a relação entre a hierarquia social urbana e a oferta de serviços de saúde na Região Metropolitana de Belo Horizonte, localizada no estado de Minas
Gerais, em 2010. Mais especificamente, analisamos em que medida os equipamentos de saúde e os médicos alocados nos serviços se distribuíam seguindo ou não a lógica da segregação socioespacial. Depois, apresentamos os detalhes da metodologia seguindo os três "problemas metodológicos" da análise da segregação urbana de Préteceille (2004), a saber, as categorias, os recortes dos espaços urbanos e os métodos estatísticos utilizados.

\section{Quais categorias?}

Para a hierarquia social urbana, escolhemos as categorias sócio-ocupacionais - Cats, utilizadas originalmente no Brasil por Préteceille e Ribeiro (1999). Seu pressuposto é o de traduzir, por meio do agrupamento de ocupações, a estrutura da divisão social do trabalho, fornecendo coerência estatística e cognitiva às diferentes posições sociais relativamente homogêneas da hierarquia social (Barcellos e Mammarella, 2001). Essa classificação já foi amplamente utilizada com base nos dados dos censos demográficos do IBGE, inclusive de forma inaugural por Mendonça (2003), para análise da segregação urbana na RMBH nos anos de 1980 e 1991. Neste artigo, realizamos uma adaptação das Cats para que seu uso fosse possível através do Censo Demográfico 2010. Em função do número elevado de categorias criadas, 24 ao todo, fizemos uma agregação em seis grupos. ${ }^{3}$

Para a identificação da oferta de serviços, escolhemos o número de estabelecimentos públicos de saúde disponível no Cadastro Nacional de Estabelecimentos de Saúde (CNES), do Ministério da Saúde, de dezembro de 2010. A razão de considerar apenas os 
estabelecimentos públicos deve-se à sub-representação do setor privado não conveniado ao SUS no cadastro. Dentre os serviços públicos, destacamos separadamente as Unidades Básicas de Saúde (UBS), por representarem os equipamentos de saúde que supostamente deveriam estar mais próximos das comunidades, constituindo-se como elo essencial e primário entre os cidadãos e a saúde pública. Quanto à verificação da oferta de estabelecimentos de saúde privados, utilizamos os dados da Relação Anual de Informações Sociais (Rais), do Ministério do Trabalho e Emprego, de 2010, relativos aos estabelecimentos empregadores em atividades econômicas da área da saúde. ${ }^{4}$

Além dos equipamentos, identificamos 0 número de médicos, obtido pela contagem de equivalentes em tempo completo, chamado na literatura específica de Full Time EquivalentFTE (Pong e Pitblado, 2002). Para tanto, cada 40 horas semanais de trabalho médico foram consideradas como equivalentes ao trabalho de um único profissional. Esse cálculo foi feito apenas para os médicos em estabelecimentos públicos, tendo em vista que o critério captura a carga horária registrada em qualquer tipo de vinculação médica, seja ela baseada em vínculos formais de trabalho ou em vínculos frouxos, incluindo aqueles relativos a procedimentos. Por essa via, a Rais não foi utilizada para cômputo de médicos exatamente por se restringir aos vínculos formais de emprego, os quais, em geral, são o formato menos frequente de contratação de médicos em serviços privados. De fato, atualmente, não é possível dimensionar o número efetivo de médicos na Saúde Suplementar através das bases de dados disponíveis.

\section{Quais recortes dos espaços urbanos?}

A unidade geográfica definida para este estudo foi a Área de Ponderação (AP), utilizada pelo IBGE para efeitos de calibração da amostra do censo demográfico. ${ }^{5}$ Trata-se de um recorte que permite representar o espaço da RMBH em torno dos atributos de interesse para análise. No caso da hierarquia social urbana, a AP é a única unidade capaz de assinalar a ocupação dos entrevistados no interior dos municípios mais populosos, já que essa informação não está disponível por setor censitário. Embora seja um recurso estatístico, a definição dessas áreas é feita em conjunto com as administrações municipais, permitindo desenhar espaços que guardam certa semelhança socioeconômica. Nesse sentido, refere-se a uma escala com nível de correspondência à prática social que se deseja analisar, no caso a segregação medida pelo critério sócio-ocupacional. Em relação aos equipamentos de saúde e médicos, o uso da AP permite superar certos limites colocados quando se consideram analiticamente as fronteiras administrativas de ação e organização das redes de atenção do SUS, delimitadas em torno dos municípios e dos distritos sanitários. Quanto às regiões metropolitanas, deve-se atentar para o fato de que a distribuição dos serviços segue determinações que não adotam essas delimitações, muitas vezes em função da conurbação desses espaços ou das desigualdades presentes nos espaços intramunicipais.

De acordo com o Censo Demográfico, em 2010, a RMBH contava com 189 AP distribuídas em 34 municípios, e apenas 16 municípios possuíam mais de uma área. No caso dos outros 18, todo o espaço municipal era considerado uma AP. Os dados referentes às Cats foram 
coletados diretamente nos microdados do censo para cada área. A contagem de estabelecimentos de saúde e de médicos não se deu de forma direta, pois os microdados do CNES e da Rais não estão disponíveis nesse nível geográfico. Para tanto, procedemos à extração das coordenadas geográficas com base nos endereços dos estabelecimentos de saúde disponíveis nos microdados e, em seguida, compatibilizamos com a malha geográfica do IBGE. A extração das coordenadas geográficas foi feita por meio de geocodificação através dos servidores da Google Inc., pelo software SIG Google Earth, e a correção de possíveis imprecisões no software QGIS versão 2.8.

\section{Quais métodos estatísticos?}

Na definição dos métodos estatísticos, levamos em consideração o objetivo de, por um lado, descrever a distribuição da hierarquia social e da oferta de serviços de saúde e de médicos no espaço do RMBH e, por outro lado, analisar a associação entre eles. Em ambas as situações, utilizamos como unidade analítica as áreas de ponderação e calculamos, para cada uma delas, as proporções de pessoas nas seis Cats, agrupadas neste trabalho, e a razão de equipamentos de saúde e de médicos por mil habitantes. Empregamos ainda, como medida descritiva de concentração, o índice de segregação (Préteceille, 2004), o qual compara a distribuição das variáveis no interior das áreas de ponderação à mesma distribuição observada na totalidade da região metropolitana. Trata-se de uma razão cujos valores acima de um $(1,0)$ descrevem a concentração relativa do atributo em questão no espaço da AP, comparativamente ao espaço da RMBH. Para analisar a associação entre segregação socioespacial e oferta de serviços de saúde, procedemos à Correlação de Pearson (Agresti e Finlay, 2012), pela qual cada categoria sócio-ocupacional (medida em proporção) foi correlacionada separadamente com cada tipo de oferta de serviços de saúde (medida em razão por mil habitantes). Assim, a proporção de uma determinada CAT foi utilizada para prever a razão de um determinado serviço de saúde por mil habitantes e vice-versa.

Por fim, optamos por verificar a possibilidade de haver correlação espacial entre os elementos destacados, tendo em vista nossa escolha pelo recorte espacial das áreas de ponderação. Nesse sentido, buscamos examinar se o comportamento dos dados é influenciado pela localização. Dessa forma, identifica-se a associação entre uma variável em uma determinada área, relativamente às áreas vizinhas, o que pode revelar padrões estatisticamente aleatórios ou correlacionados (Marques, Scalon e Oliveira, 2008). Objetivamente, a correlação espacial é uma associação linear na qual se comparam os valores da variável de interesse em uma localidade com a média dessa variável nas localidades vizinhas (Almeida e Guanzirole, 2013). A forma mais comum de mensurar a correlação espacial, utilizada neste trabalho, é o Índice de Moran Global, cujos valores oscilam de -1 a 1 , sendo, quanto mais próximo de zero (0), menor a correlação espacial existente. Complementarmente, utilizamos o Índice de Moran Local, buscando evitar inconsistências do índice global, que não reflete padrões locais de correlação espacial (Anselin, 1995). 


\section{Distribuição sócio-ocupacional e de serviços de saúde na $\mathrm{RMBH}$}

Em 2010, a população da RMBH era composta de 4.883 .970 habitantes, dos quais $49,6 \%$ estavam ocupados na semana de referência do Censo Demográfico (o que representava $57,2 \%$ da população em idade ativa e $92,8 \%$ da população economicamente ativa). Como mostra a Tabela 1, 12,5\% da população ocupada constituía o estrato mais alto da hierarquia social, o correspondente aos empregadores, dirigentes e profissionais de nível superior. $\mathrm{Na}$ sequência da hierarquia, aparecia o estrato de ocupações não manuais de rotina, o mais expressivo, com $36,2 \%$ da população ocupada. 0 grupo era composto de trabalhadores em ocupações artísticas, técnicas, de escritório, do comércio, dos serviços de proteção e segurança, entre outras. Já a categoria de ocupações manuais em atividades dos serviços perfazia $25,9 \%$ e a de ocupações manuais em atividades da indústria, 18,5\%. A categoria de trabaIhadores rurais apresentou-se como a menos expressiva, com 1,2\%. Ressalta-se, ainda, que $5,3 \%$ estavam registrados no Censo em ocupações mal definidas.

A análise dos índices de segregação também é apresentada, na Tabela 1, por meio do número e da proporção de Áreas de Ponderação que obtiveram valor maior que 1,0 , isto é, que possuíam uma representação de ocupados, na categoria correspondente, superior ao observado na totalidade da RMBH. A proporção que concentrava o estrato de empregadores, dirigentes e profissionais de nível superior era a menor, $21,1 \%$, o equivalente a 40 das 189 unidades. Esse valor pode ser explicado, em parte, pela frequência de pessoas na categoria, mas também pode indicar a existência de segregação socioespacial. À medida que a hierarquia social avança para os outros estratos, que poderiam ser identificados como grupos intermediários e inferiores da estrutura social, o número de áreas aumenta. Os trabalhadores em ocupações não manuais de rotina se concentravam em $36,5 \%$ das áreas, os manuais dos serviços, em $64 \%$, e os manuais da indústria, em $64,6 \%$. Note-se que não necessariamente 0 número de ocupados em cada estrato influenciava nas proporções, pois a CAT 2 tinha uma relação de 880.995 pessoas para 69 áreas, enquanto a CAT 3, de 629.379 para 121 e a CAT4, de 449.476 para 122. Assim, as proporções refletiam, sobretudo, a existência de segregação no espaço metropolitano para os dois grupos de ocupações manuais, tendo em vista que 0 conjunto dos não manuais de rotina estava mais disperso.

A Figura 1 mostra mapas comparativos das distribuições das seis Cats por área de ponderação. Observa-se que o estrato mais elevado se concentrava, principalmente, na região Centro-Sul de Belo Horizonte, a qual se constituiu historicamente como uma região nobre, mas também em bairros como Concórdia, União e Ipiranga, na região Nordeste; Padre Eustáquio e Dom Cabral, na região Noroeste; Nova Suíça, Grajaú e Buritis, na região Oeste; e Santa Amélia, Paquetá e Ouro Preto, na região da PampuIha. Algumas áreas isoladas dos municípios de Contagem, Nova Lima e Betim também apareciam com relevância. Parte desses bairros citados também agrupava ocupações não manuais de rotina, mas estas avançavam principalmente para outras localidades dos municípios de Belo 
Tabela 1 - Distribuição da população ocupada, por categoria sócio-ocupacional, estabelecimentos de saúde, médicos e Áreas de Ponderação com índice de segregação acima de 1,0. RMBH, 2010

\begin{tabular}{|c|c|c|c|c|c|}
\hline \multirow{2}{*}{ Categorias sócio-ocupacionais (Cats) } & \multirow[t]{2}{*}{$\mathbf{N}$} & \multirow{2}{*}{$\begin{array}{l}\text { \% em relação à } \\
\text { pop. ocupada }\end{array}$} & \multicolumn{2}{|c|}{$\begin{array}{c}\text { AP c/ índice de } \\
\text { segregação superior a 1,0 }\end{array}$} & \multirow{2}{*}{$\begin{array}{l}\text { Moran } \\
\text { Globa }\end{array}$} \\
\hline & & & $\mathbf{N}$ & $\%$ & \\
\hline $\begin{array}{l}\text { CAT } 1 \text { - Empregadores, dirigentes e } \\
\text { profissionais de nível superior }\end{array}$ & 305.052 & 12,5 & 40 & 21,2 & 0,1988 \\
\hline CAT 2 - Não manual de rotina & 880.995 & 36,2 & 69 & 36,5 & 0,1476 \\
\hline CAT 3 - Manual em atividades de serviços & 629.379 & 25,9 & 121 & 64,0 & 0,0399 \\
\hline CAT 4 - Manual em atividades da indústria & 449.476 & 18,5 & 122 & 64,6 & 0,0820 \\
\hline CAT 5 - Trabalhadores rurais & 39.713 & 1,6 & 56 & 29,6 & 0,0760 \\
\hline CAT 6 - Ocupações mal definidas & 128.635 & 5,3 & 92 & 48,7 & 0,0633 \\
\hline \multirow[t]{2}{*}{ Oferta de serviços de saúde } & \multirow[t]{2}{*}{$\mathbf{N}$} & \multirow{2}{*}{$\begin{array}{l}\text { Razão por mil } \\
\text { habitantes }\end{array}$} & \multicolumn{2}{|c|}{$\begin{array}{c}\text { AP c/ índice de } \\
\text { segregação superior a 1,0 }\end{array}$} & \multirow{2}{*}{$\begin{array}{l}\text { Moran } \\
\text { Globa }\end{array}$} \\
\hline & & & $\mathrm{N}$ & $\%$ & \\
\hline Estabelecimentos públicos & 978 & 0,20 & 78 & 41,3 & 0,0111 \\
\hline Estabelecimentos privados & 7.632 & 1,56 & 21 & 11,1 & 0,0407 \\
\hline Unidades Básicas de Saúde & 613 & 0,13 & 84 & 44,4 & 0,0306 \\
\hline Médicos em estabelecimentos públicos & 10.496 & 2,15 & 35 & 18,5 & $-0,0036$ \\
\hline Médicos em UBS & 2.391 & 0,49 & 78 & 41,3 & 0,0504 \\
\hline População residente & 4.883 .970 & & & & \\
\hline População ocupada & 2.433 .251 & & & & \\
\hline Áreas de Ponderação & 189 & & & & \\
\hline
\end{tabular}

Fonte: elaboração própria, a partir dos dados do Censo Demográfico, Cadastro Nacional de Estabelecimentos de Saúde e Relação Anual de Informações Sociais.

Horizonte. Destacam-se os bairros Carlos Prates e Caiçara, na região Noroeste; Santa Inês, Boa Vista e São Geraldo, na divisa com Sabará; e Santa Terezinha, Serrano e Alípio de Melo, na divisa com Contagem. Os não manuais de rotina também se estendiam para o município de Contagem, principalmente na região do bairro Nacional, na divisa com a região da Pampulha, e na região do Jardim Riacho, na fronteira com a região do Barreiro.
Para as Cats 3 e 4, de trabalhadores manuais, observamos que a concentração se distanciava mais do centro, deixando de ter relevância nas áreas da capital que estavam segregadas segundo a CAT 1 e passando a se concentrar em outras regiões da capital e em outros municípios da RMBH. Entre os manuais do setor de serviços, o agrupamento era percebido, em BH, nas regiões do Barreiro (bairros Tirol, Jatobás e Lindeia); Venda Nova (Jardim dos 
Comerciários e Justinópolis); Nordeste (Ribeiro de Abreu, Paulo IV e Capitão Eduardo); e Leste (Taquaril, Granja de Freitas, Santa Inês, Boa Vista e São Geraldo), e nos municípios Nova Lima, Esmeraldas, Lagoa Santa, Sabará e Vespasiano. Já os manuais em atividades industriais se mostravam de forma ainda mais periférica, incluindo os municípios de Contagem (bairros Nacional, Nova Contagem e Vargem das Flores), Juatuba, Igarapé, São Joaquim de Bicas, Betim, Pedro Leopoldo, São José da Lapa, Caeté e lbirité.

Os trabalhadores rurais estavam mais representados nas extremidades da $\mathrm{RMBH}$, com destaque para os municípios de Caeté, Itaguara, Rio Manso, Brumadinho, São Joaquim de Bicas, Esmeraldas, Jaboticatubas e Baldim, e também de forma expressiva em Taquaraçu de Minas, Sarzedo, Mario Campos, Betim, Florestal, Igarapé e Itatiaiuçu. Em relação à CAT 6, de ocupações mal definidas, a distribuição assemelhava-se à da CAT 2, de ocupações não manuais de rotina, enfatizando que esse grupo populacional, o maior entre os elencados, poderia ser ainda mais numeroso, aproximando-se de quase um milhão de pessoas ou $40 \%$ da força de trabalho ocupada.

Como demonstrado por Mendonça e Marinho (2015), a estrutura da RMBH organiza-se através de anéis, em que os grupos superiores se localizam nas áreas centrais, e os grupos mais operários e populares tornam-se mais evidentes nas periferias. Essa disposição reflete o desenvolvimento histórico da região, a qual foi (e ainda é) fortemente marcada pela ação do Estado em torno de investimentos, principalmente, em infraestrutura e em logística que objetivaram o crescimento econômico da metrópole. Os autores destacam que a estrutura observada em 2010 seguiu as tendências existentes pelo menos desde a década de 1980, com permanência da conformação de anéis, embora a composição social tenha ficado mais complexa e mesclada. Evidencia-se que se acentuou a transformação das áreas centrais em torno de estratos sociais superiores, ou "aburguesamento", especificamente nas regiões do Barreiro, em Belo Horizonte, e do bairro Eldorado até a região central da cidade de Contagem e no centro de Betim.

Em relação aos serviços de saúde, foram identificados 8.610 estabelecimentos, sendo 7.632 privados, identificados na Rais, e 978 públicos, constatados no CNES (Tabela 1). Também foram contadas as Unidades Básicas de Saúde (UBS), que somavam 613. Quando verificada a concentração dos serviços no espaço, o índice de segregação mostrou que os estabelecimentos privados eram mais representativos em 21 $(11,1 \%)$ áreas de ponderação, em comparação à totalidade da RMBH. Já os estabelecimentos públicos, em 78 (41,3\%), e as UBS, em 84 $(44,4 \%)$. Segundo a Figura 2 , os equipamentos privados concentravam-se na área hospitalar e na região da avenida do Contorno, em Belo Horizonte, e pontualmente na região do Barreiro e no bairro Belvedere. Também se apresentava com alguma expressividade na região do bairro Eldorado, em Contagem, e no Centro de Betim. Note-se que essas áreas são as que seguiram a tendência de aburguesamento observada por Mendonça e Marinho (ibid.).

Já os serviços públicos e as Unidades Básicas de Saúde se espalhavam mais pelo espaço, configurando distribuições semelhantes - o que não é por acaso, uma vez que as UBS representavam mais da metade dos estabelecimentos públicos. Essa é uma tendência 
Figura 1 - Mapas comparativos das distribuições das categorias sócio-ocupacionais por área de ponderação - RMBH, 2010
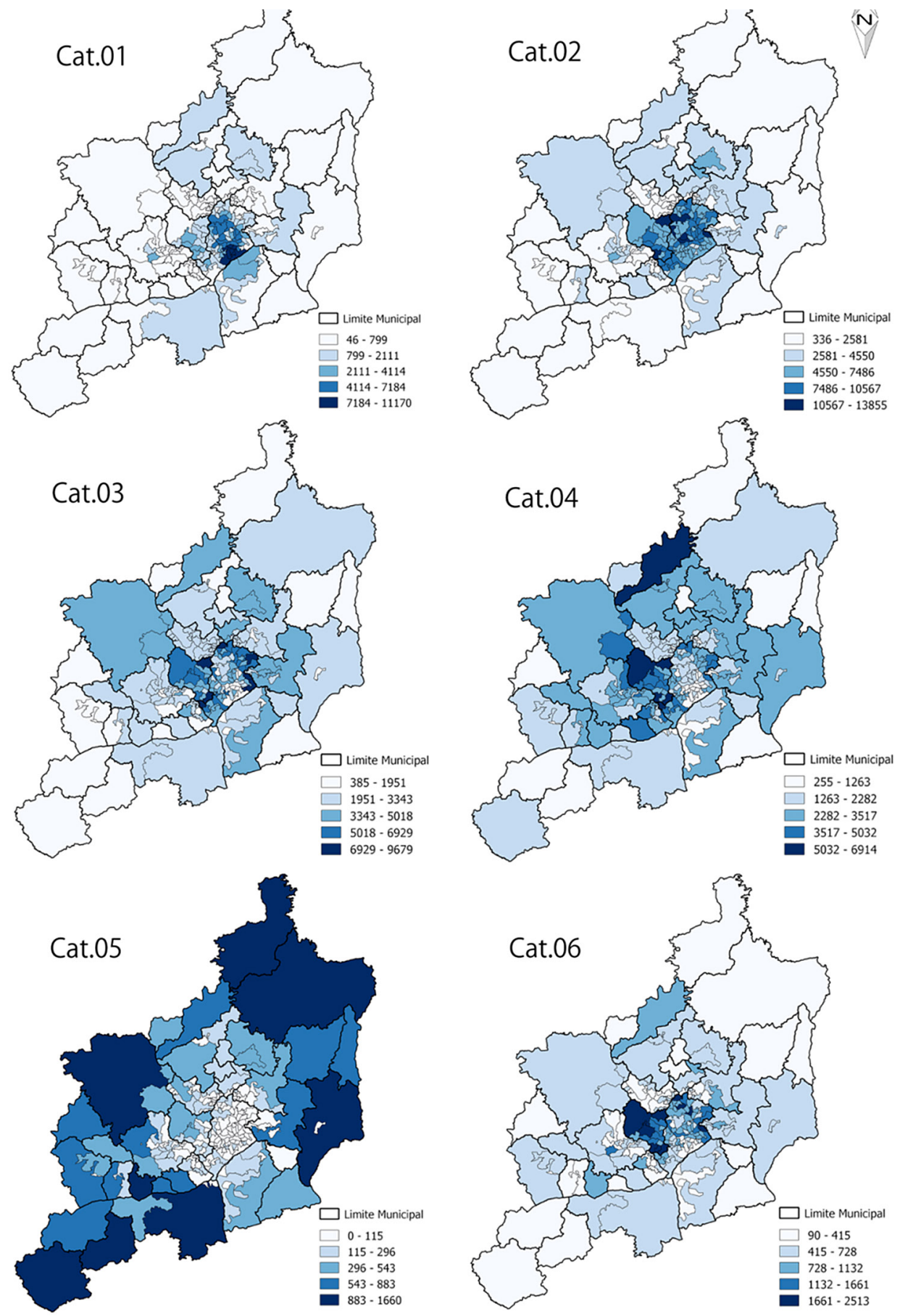

Fonte: elaboração própria, a partir dos dados do Censo Demográfico de 2010. 
observada em todo o Brasil, sobretudo, desde a criação da Estratégia de Saúde da Família (ESF), a principal política responsável pela interiorização da saúde no País nas últimas décadas (Girardi et al., 2016). Na RMBH, a maior presença desses equipamentos foi verificada nos bairros Carlos Prates, Padre Eustáquio e Caiçara, na região Noroeste de Belo Horizonte, e nos bairros Horto e Sagrada Família, na região Leste, também da capital. Em Contagem, sobressaíram-se os bairros Nacional, Nova Contagem e Várzea das Flores. Citam-se ainda várias áreas nos municípios Pedro Leopoldo, Sabará, Caeté, Lagoa Santa, Santa Luzia, Ibirité, Brumadinho, Esmeraldas, Ribeirão das Neves e Nova Lima.

Em relação aos médicos, foram assinalados 10.496 profissionais (em equivalência de 40 horas - FTE) em estabelecimentos públicos, sendo 2.391 em UBS (Tabela 1). Quando observamos pela ótica do espaço, $18,5 \%$ das áreas de ponderação tinham uma razão de médicos por mil habitantes superior à da RMBH. Essa proporção sobe para $41,3 \%$ no caso dos médicos em UBS, os quais eram mais representativos nos bairros Floramar, Heliópolis e São Bernardo da região Norte de BH, nos bairros Taquaril e Granja de Freitas da região Leste, na região do Barreiro e nos municípios de Ibirité e Nova Lima. Note-se que, embora os equipamentos públicos estivessem mais bem distribuídos do que os equipamentos privados, o mesmo não se pode dizer em relação aos médicos alocados nos serviços próprios do SUS. Nossa hipótese é a de que os médicos tendem a se concentrar nos serviços privados de média e alta complexidade e nos consultórios particulares, em geral, levando à desassistência ou à insuficiência desses profissionais na atenção primária à saúde como demonstraram alguns estudos (Campos, Machado e Girardi, 2009; Girardi et al., 2016). Os limites das bases de dados sobre oferta de recursos humanos em saúde, porém, não permitem que essa hipótese seja testada neste estudo.

Os valores do Índice de Moran Global destacam uma baixa correlação espacial para as Cats 1 e 2 e uma correlação praticamente inexistente para as demais categorias sócio- ocupacionais e para as diferentes ofertas de serviços de saúde. Segundo a Tabela 1, o valor do índice foi de 0,1988 e 0,1476, respectivamente para as duas primeiras Cats. Nas demais ocorrências, os valores foram inferiores a 0,1. De maneira geral, portanto, os resultados do índice mostraram o comportamento aleatório dos dados quando distribuídos segundo áreas de ponderação, à exceção dos estratos de empregadores e profissionais de nível superior e ocupações não manuais de rotina, que apresentam baixa segregação no espaço.

Tais resultados destoam da análise do índice de segregação e dos mapas realizada acima, a qual indica a existência de segregação tanto das categorias sócio-ocupacionais, quanto da oferta de serviços de saúde. Supomos, por um lado, que a escolha de utilizar as áreas de ponderação como unidade de análise espacial pode ter afetado o comportamento dos dados e, nesse sentido, o exame por essa via não refletiria a realidade da divisão social do espaço da metrópole em pauta. Por outro lado, ao contrário, o resultado pode indicar uma particularidade no padrão de distribuição social da RM$\mathrm{BH}$, a qual ocorreria de forma não contígua e heterogênea, pelo menos se considerarmos os limites geográficos das áreas de ponderação.

Em estudo que comparou as estruturas sociais das regiões metropolitanas do Rio de 
Figura 2 - Mapas comparativos da distribuiç̧ão de serviços de saúde e de médicos, em FTE*, por área de ponderação - RMBH, 2010

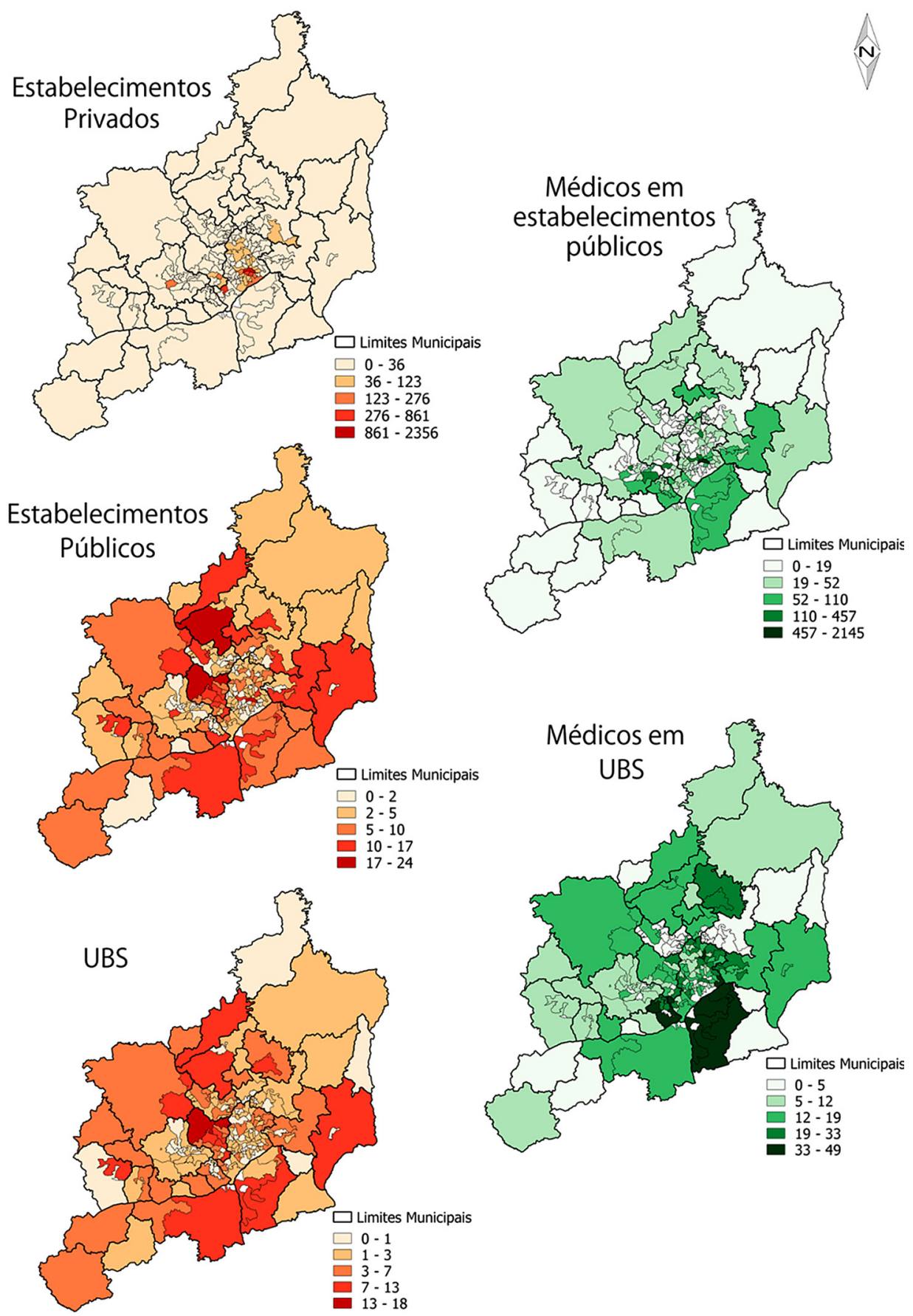

Fonte: elaboração própria, a partir dos dados do Cadastro Nacional de Estabelecimentos de Saúde e da Relação Anual de Informações Sociais.

* Full Time Equivalent. cada 40 horas semanais de trabalhos médicos em serviços de saúde corres-pondem a um médico. 
Janeiro e de São Paulo, Marques, Scalon e Oliveira (2008) também lançaram mão de áreas de ponderação e análise de correlação espacial via Índice Moran. Opostamente aos nossos resultados, os autores demonstraram um padrão altamente segregado dos estratos sociais no espaço das duas regiões (com valores de Moran Global próximos a 0,80), indicando também similaridade entre elas em relação à distribuição dos indivíduos no interior das áreas de ponderação. Para testar a possibilidade de um padrão diferenciado na RMBH, no entanto, pesquisas deverão ser feitas em estudos futuros à luz de outros níveis de abrangência geográfica, utilizando outras categorias sociais, no sentido de verificar alterações de comportamento dos dados, especialmente, em áreas geográficas menos desagregadas.

\section{Associação entre segregação socioespacial e oferta de serviços de saúde}

A Tabela 2 apresenta o resultado de correlações simples entre as variáveis de hierarquia social e as variáveis de oferta de serviços de saúde. Cada categoria sócio-ocupacional (em proporção) foi correlacionada, separadamente, com cada tipo de oferta de serviços de saúde (em razão por mil habitantes). No caso da oferta de serviços privados, verificaram-se correlações positivas e estatisticamente significantes com as Cats 1 e 2, sendo uma correlação de média a forte com a primeira $(0,427)$ e fraca com a segunda $(0,157)$. Note-se que, enquanto $11,1 \%$ das áreas de ponderação da $\mathrm{RMBH}$ concentravam esse tipo de serviço, naquelas áreas em que prevaleciam os empregadores, dirigentes e profissionais a proporção era de $50 \%$ e, nas que prevaleciam as ocupações não manuais de rotina, $18,8 \%$. Portanto, os dados sugerem uma associação entre localização territorial do mercado privado de atenção à saúde e áreas segregadas por populações de estratos superiores da hierarquia social. Tanto os serviços privados quanto as Cats em questão, sobretudo a primeira, concentravam-se no município de Belo Horizonte, com destaque para os bairros da região Centro-Sul, e pontos isolados de Contagem e Betim. A associação entre oferta de serviços privados de saúde e níveis superiores na hierarquia social fica ainda mais evidente quando se observam correlações negativas com as Cats de trabalhadores manuais em atividades dos serviços $(-0,370)$ e trabalhadores manuais em atividades da indústria $(-0,330)$.

A oferta de serviços públicos de saúde, por seu turno, segue uma lógica quase oposta. À medida que se caminha pela hierarquia social, da categoria superior até a inferior, a proporção de áreas que concentram equipamentos públicos aumenta. Enquanto as Cats 1 e 2 têm correlações negativas com esses serviços, de $-0,163$ e -0,304, respectivamente, as Cats 4 e 5 têm correlações positivas, de 0,204 e 0,423. Esse padrão é ainda mais manifesto quando se analisa a distribuição de Unidades Básicas de Saúde, já que as correlações para as duas primeiras categorias sócio-ocupacionais passam para -0,294 e -0,457 e as das duas últimas para 0,310 e 0,504. A CAT 3, que não mostrou correlação com os estabelecimentos públicos, passa a apresentar um valor positivo de 0,152 na análise que isola as UBS. Os resultados apontam que os serviços públicos de saúde, sobretudo os de atenção primária, intensificavam-se 
em áreas de grupos sócio-ocupacionais inferiores na hierarquia social. É interessante ressaltar também que as áreas segregadas pelo estrato dos empregadores, dirigentes e profissionais de nível superior estavam em situação um pouco melhor do que as áreas de ocupações não manuais de rotina, no que se refere à oferta de serviços públicos, ainda que ambas tendessem a maior presença de estabelecimentos privados. Muito provavelmente, isso ocorre em razão de a área hospitalar localizada na região Centro-Sul de Belo Horizonte ter uma função estratégica para o SUS e reunir um conjunto expressivo de equipamentos públicos, que atendem não só aos moradores de $\mathrm{BH}$, mas aos de todo o estado.

Por fim, a oferta de médicos em serviços públicos não apresentou correlação estatisticamente significante com nenhum estrato da hierarquia social, embora os testes tenham mostrado valores positivos com as Cats 1 e 2 e negativos com as demais. Apenas a oferta de médicos em UBS mostrou uma fraca correlação positiva, de 0,169, com o estrato manual em atividades da indústria. Note-se, no entanto, que, em uma correlação realizada à parte, a oferta de médicos em serviços públicos apresentou uma correlação alta, de 0,529, com a oferta de estabelecimentos privados, demonstrando uma preferência dos profissionais a se ocuparem em serviços públicos quando estes estão em áreas que concentram o mercado privado de saúde.

A distribuição de médicos no espaço, nesse sentido, segue lógica distinta, pois não depende apenas da existência de equipamentos, mas de aspectos que vão desde as escoIhas pessoais em se deslocar para trabalhar em determinadas localidades até a existência de estratégias de atração e fixação por parte dos agentes contratantes dessa força de trabaIho. Este tem sido um problema persistente no Brasil, particularmente, na gestão municipal da atenção primária à saúde (Girardi et al., 2011).

Tabela 2 - Resultados das correlações entre categorias sócio-ocupacionais e serviços de saúde - RMBH, 2010

\begin{tabular}{|c|c|c|c|c|c|}
\hline \multirow[b]{2}{*}{ Categorias sócio-ocupacionais } & \multicolumn{5}{|c|}{ Serviços de saúde } \\
\hline & $\begin{array}{l}\text { Estab. } \\
\text { Privados }\end{array}$ & $\begin{array}{l}\text { Estab. } \\
\text { Públicos }\end{array}$ & UBS & $\begin{array}{l}\text { Médicos } \\
\text { Públicos }\end{array}$ & $\begin{array}{l}\text { Médicos } \\
\text { UBS }\end{array}$ \\
\hline $\begin{array}{l}\text { CAT } 1 \text { - Empregadores, dirigentes e profissionais de } \\
\text { nível superior }\end{array}$ & $0,427^{* *}$ & $-0,163^{*}$ & $-0,294^{* *}$ & 0,059 & $-0,14$ \\
\hline CAT 2 - Não manual de rotina & $0,157^{*}$ & $-0,324^{* *}$ & $-0,457^{* *}$ & 0,115 & $-0,131$ \\
\hline CAT 3 - Manual em atividades de serviços & $-0,370^{* *}$ & $-0,002$ & $0,152^{*}$ & $-0,079$ & 0,123 \\
\hline CAT 4 - Manual em atividades da indústria & $-0,330^{* *}$ & $0,204^{* *}$ & $0,310^{* *}$ & $-0,077$ & $0,169^{*}$ \\
\hline CAT 5 - Trabalhadores rurais & $-0,083$ & $0,423^{* *}$ & $0,504^{* *}$ & $-0,045$ & 0,055 \\
\hline
\end{tabular}

Fonte: elaboração própria, a partir dos dados do Censo Demográfico, Cadastro Nacional de Estabelecimentos de Saúde e Relação Anual de Informações Sociais.

* Correlação significante a 0,05 (2 caudas).

**Correlação significante a 0,01 (2 caudas). 
Uma ação recente do Governo Federal para provimento de médicos em municípios brasileiros, o "Programa Mais Médicos", tem mostrado alguma eficiência em contornar a ausência ou a insuficiência de médicos em áreas de maior necessidade. No entanto, permanecem expressivas desigualdades distributivas e insegurança assistencial devido à dependência que alguns municípios passaram a ter em relação a esse provimento (ibid., 2016).

Os resultados apresentados corroboram o estudo de Torres e Bichir (2007) que demonstrou uma importante cobertura de infraestrutura urbana e acesso a políticas públicas de saúde em áreas pobres e periféricas da cidade de São Paulo. Os autores constataram, ainda, a existência de variações significativas no acesso aos serviços de saúde que se explicavam pelo espaço, já que as áreas centrais tendiam a ter melhores condições, mesmo com expressiva oferta de equipamentos em áreas pobres e periféricas. Para eles, isso pode ser explicado, entre outros fatores, pelo fato de haver decréscimo populacional nas áreas centrais, o que acarreta menores demandas de saúde. Além disso, a concentração de serviços especializados no centro limita as condições de acesso dos moradores que dependem do SUS. Além disso, nas áreas pobres, sobretudo em favelas, a "pressão demográfica" sobre os equipamentos de saúde é maior, e tal situação se intensifica pela alta rotatividade de médicos nas unidades de saúde dessas regiões. Outros fatores não desprezíveis são destacados para explicar as diferenças de acesso, como os atributos individuais da população (sexo, renda, raça, escolaridade), as decisões estatais que visam ou não a ampliar o acesso a determinados serviços públicos e a ação dos movimentos sociais e das redes provenientes de laços familiares e comunitários.

De forma geral, a ação do Estado no espaço tende a se direcionar para áreas de maior necessidade de saúde, nas quais se localiza a população dependente dos serviços públicos que não pode pagar por atendimentos na rede privada. Tal ação se coaduna aos princípios de equidade e igualdade do SUS, justificando a priorização da oferta de serviços e ações de saúde em espaços cuja população residente enfrenta maiores riscos de adoecimento e óbito devido a desigualdade de renda, bens e serviços existente na realidade brasileira (Vasconcelos e Pasche, 2008). Essa focalização, porém, pode reforçar a posição inferior desses grupos, na medida em que o Estado atua conformando divisões por status no espaço, as quais explicitam e reforçam o exercício do poder entre grupos sociais e delineiam relações assimétricas (Marcuse, 2004). De fato, são muito comuns políticas públicas que, ao invés de diminuírem as desigualdades sociais no espaço urbano, reforçam o status inferior de grupos marginalizados, estigmatizados e desassistidos, ainda que proporcionem melhorias em suas condições de vida (Préteceille, 2004).

No caso das políticas de saúde no Brasil, essa situação é particularmente problemática, tendo em vista o contexto atual de municipalização e regionalização das redes de atenção à saúde. Essas redes estão organizadas tendo como porta de entrada os serviços de atenção primária, cuja alocação no espaço é de responsabilidade dos entes municipais. À medida que os serviços ampliam a complexidade, a alocação passa a ser feita também pelos entes estaduais e federais, apresentando uma configuração inevitavelmente concentrada em municípios mais 
ricos, devido à demanda por mais tecnologia e investimentos a conjuntos populacionais maiores. Tal configuração define uma distribuição espacial concentrada dos serviços de média e alta complexidade (Rodrigues, Amaral e Simões, 2007), o que ocorre acompanhando a alocação dos serviços privados, como é observado principalmente na área hospitalar, na região Centro-Sul de Belo Horizonte. Essa tendência pode ser explicada pela alta dependência do SUS em relação aos hospitais e clínicas especializadas privadas, já que a oferta pública em equipamentos próprios é insuficiente para atender à demanda (Paim et al., 2011).

No que diz respeito ao mercado privado de saúde, não é novidade que a cobertura dos planos de saúde depende da capacidade de financiamento dos indivíduos e dos grupos. Esta é uma explicação importante das desigualdades de utilização dos serviços de saúde, já que os portadores de planos têm maior probabilidade de utilização do que os que não possuem (Travassos et al., 2000). Nesse sentido, a distribuição desses serviços no espaço tende a se direcionar para municípios mais ricos e com maior densidade demográfica. Além disso, tais serviços em geral são mais diversificados, conformando uma rede própria que opera sem conhecer os limites geopolíticos e ou de ação do SUS. Isso é notório quando são observados os fluxos de clientes dos planos de saúde, verificando-se que esses mercados são ainda mais amplos do que as fronteiras geopolíticas das regiões metropolitanas (Andrade et al., 2012).

A concentração dos serviços privados de saúde na RMBH não está dissociada do capitalismo e dos mercados que se formam através dele. 0 espaço urbano formado pela iniciativa privada, com o apoio dos agentes políticos, produz hierarquias com desvantagens para as classes sociais descapitalizadas (Harvey, 2004). Uma característica desse espaço é a concentração de determinado tipo de serviço em áreas definidas por uma identidade própria. Na RMBH (e em outras grandes metrópoles), é o caso da área hospitalar. Em termos da reprodução e expansão dos estabelecimentos privados de saúde, a proximidade dos serviços é benéfica, uma vez que facilita os itinerários terapêuticos entre estabelecimentos. 0 usuário da rede privada tem seus percursos facilitados pela possibilidade de realizar diversos procedimentos com pequenos deslocamentos dentro de uma mesma área e a uma distância próxima de seu local de residência.

Além disso, a concentração dos equipamentos privados, os quais também são largamente utilizados pelo SUS para superar a oferta insuficiente de serviços, gera uma desigualdade contínua no espaço. Novos estabelecimentos optarão por se instalarem nas regiões onde há fluxo de pacientes e na região conhecida pela oferta de serviços de saúde. Do ponto de vista do planejamento urbano, a concentração desses serviços é uma "via de mão dupla", uma vez que a cidade cria uma região especializada em serviços de saúde, mas, ao mesmo tempo, gera desigualdade na oferta. Por mais que os serviços do SUS, seja através de equipamentos próprios ou por meio de convênio com a rede privada, estejam dispostos nessas localidades de forma coerente e sejam mobilizados para garantir a utilização democrática de diversos recursos, as distâncias sociais são reafirmadas pela presença de segregação espacial. Com efeito, os percursos do usuário do sistema público tendem a seguir movimentos de vivência prolongada da experiência territorial no curso 
das tentativas de tratamento, o que se faz sentir tanto pelas longas distâncias percorridas, quanto pelas descontinuidades nos atendimentos, reforçando a posição inferior desses indivíduos no espaço social.

Um último aspecto a ser tratado neste estudo refere-se à análise de correlação feita pelo Índice de Moran Local, na qual encontramos resultados semelhantes aos do Índice de Moran Global. Apenas algumas AP apresentaram correlação espacial significativa, indicando que nas demais AP a distribuição das variáveis analisadas se comporta-se de forma aleatória, isto é, sem correlação com o espaço. Entretanto, a análise descritiva dos mapas e as correlações realizadas demonstraram a existência de uma segregação dessas variáveis no espaço da RMBH. Dados esses indicadores positivos de segregação, os baixos valores dos Índices de Moran, global e local, e da significância de correlação espacial, decorre a hipótese: a delimitação territorial utilizada como base de análise econométrica espacial pode não ser a que melhor se correlaciona com a realidade distributiva das Cats, dos serviços e dos médicos. A problemática implica a identificação e o teste estatístico, em estudos futuros, de outras matrizes de peso e/ou unidades geográficas que possam ter correlações mais significativas.

De qualquer maneira, este estudo alinha-se com aqueles que têm buscado compreender o fenômeno da saúde no espaço, extrapolando os limites políticos dos municípios e microrregiões de saúde, privilegiando um olhar intraurbano (Torres, 2006; Andrade et al., 2012; Souza et al., 2016). Afinal, a pesquisa socioespacial busca uma identificação embasada na articulação entre as relações sociais e o espaço. Embora o espaço não possa ser reduzido apenas à materialidade, sua construção dá-se a partir dela (Souza, 2013). Uma vez que, no interior das metrópoles, essas articulações e relações sociais no espaço apresentam-se de forma desigual, são importantes estudos que contemplem o interior dos municípios. Incorporar o espaço como uma variável na análise de distribuição dos serviços de saúde nas unidades intraurbanas vem sendo apresentado como uma possibilidade de enriquecimento metodológico, a partir da capacidade de fornecimento de insumos para identificar e melhor compreender as lógicas aí existentes (Bousquat, Cohn e Elias, 2006).

\section{Considerações finais}

0 exercício aqui proposto foi o de compreender a organização dos serviços de saúde no espaço, investigando em que medida haveria relação com a hierarquia social e com os processos de segregação presentes. Assim, buscamos analisar em que sentido as distribuições dos serviços públicos e privados de atenção à saúde acompanham a mesma lógica da distribuição da estrutura sócio-ocupacional. Entendemos que ambos os fenômenos estão relacionados, na medida em que se conformam, ao mesmo tempo, como estruturas e produtos dos processos mais amplos de segregação social que ocorrem no espaço urbano. Assim, da mesma forma que definem divisões sociais reificadas no espaço, também são afetadas pelas relações sociais que se processam por meio das hierarquias daí derivadas.

Além disso, procuramos compreender como essa relação ocorre em uma escala 
intramunicipal, buscando superar os limites usualmente derivados das definições administrativas e de planejamento utilizados na alocação dos serviços de saúde. Para tanto, realizamos um estudo quantitativo do caso da Região Metropolitana de Belo Horizonte (RMBH) em 2010, tendo como referência geográfica as Áreas de Ponderação do IBGE e sua composição em termos sócio-ocupacionais e de oferta de equipamentos de saúde.

Do ponto de vista da conformação do espaço urbano a partir da ação organizada do Estado na prestação de serviços de assistência à saúde, na medida em que tal conformação está associada à segregação urbana, o trabalho permitiu tecer algumas hipóteses. Em primeiro lugar, localidades com maiores necessidades e adensamento demográfico, localizadas, em geral, em municípios vizinhos de Belo Horizonte e que concentram grupos sócio-ocupacionais inferiores, são preferidos na alocação de equipamentos públicos de atenção primária, ao contrário de áreas de estratos nobres e intermediários localizados principalmente na capital. Nesse sentido, os serviços são alocados em áreas com populações dependentes do SUS. Em segundo lugar, apesar de maior oferta de equipamentos, as áreas de grupos inferiores não possuem maior oferta de médicos. Nesse caso, segue-se uma lógica diferente, a qual depende fundamentalmente das escolhas dos profissionais em trabalhar nessas áreas e da existência de políticas públicas específicas que afetem essas escolhas por meio da oferta de incentivos aos profissionais.

Já a ação do mercado privado de assistência à saúde segue a lógica da segregação dos estratos superiores da hierarquia social. Assim, os equipamentos localizam-se principalmente na área mais capitalizada da metrópole, a Centro-sul de Belo Horizonte, onde está localizada a área hospitalar. Seguindo a tendência da hierarquização social das últimas décadas, esses equipamentos também se localizam naquelas áreas que passaram por processo de aburguesamento, nomeadamente as regiões do Barreiro em Belo Horizonte, do Eldorado e centro de Contagem e do centro de Betim.

0 presente trabalho permite concluir sobre a necessidade de testar outras unidades geográficas intramunicipais, como os distritos sanitários ou regiões administrativas definidas pelas gestões municipais, ou a construção de unidades que agreguem áreas de ponderação. Recomendam-se, ainda, a utilização e a comparação de diferentes categorias de análise, que expressem outras formas da organização da hierarquia social, tipos de equipamentos e de profissionais de saúde. Igualmente recomendável é a realização de estudos comparativos com outras realidades metropolitanas brasileiras. Nota-se, portanto, a existência de um campo de estudos ainda aberto a diversas possibilidades analíticas. 


\section{[I] https://orcid.org/0000-0002-5759-5039}

Universidade Federal de Minas Gerais, Faculdade de Medicina, Núcleo de Educação em Saúde Coletiva, Estação de Pesquisa de Sinais de Mercado. Belo Horizonte, MG/Brasil.

lucaswander@hotmail.com

\section{[II] https://orcid.org/0000-0003-0089-9602}

Universidade Federal de Minas Gerais, Faculdade de Medicina, Núcleo de Educação em Saúde Coletiva, Estação de Pesquisa de Sinais de Mercado. Belo Horizonte, MG/Brasil.

erickolifaria@gmail.com

\section{[III] https://orcid.org/0000-0003-4026-2390.}

Pontifícia Universidade Católica de Minas Gerais, Faculdade de Ciências Humanas, Programa de Pós-Graduação em Ciências Sociais. Belo Horizonte, MG/Brasil.

julia_pucminas@yahoo.com.br

\section{Notas}

(*) O presente artigo se fundamenta no trabalho "Estado, mercado e segregação socioespacial: o caso dos serviços de saúde na Região Metropolitana de Belo Horizonte em 2010", apresentado no IV Simpósio de Ciências Sociais da PUC Minas em outubro de 2015, como parte do GT2 Desafios globais e locais: territórios desiguais e habitat. $\mathrm{O}$ trabalho foi realizado com apoio da Coordenação de Aperfeiçoamento de Pessoal de Nível Superior (Capes) - Código de Financiamento 001 e da Fundação de Amparo à Pesquisa de Minas Gerais (Fapemig).

(1) O "Programa Mais Médicos" (PMM) foi criado em 2013 com o objetivo de ampliar a oferta de médicos no País, tem como uma de suas iniciativas a alocação emergencial de profissionais em áreas de maior necessidade. Na execução do programa, municípios e áreas intramunicipais a serem atendidos são classificados segundo determinados critérios, de maneira a caracterizar tal necessidade, e os próprios municípios manifestam o interesse em receber médicos.

(2) Optamos por não utilizar o conceito de território devido à sobreposição que às vezes acontece com a ideia de espaço. Em alguns trabalhos, os dois termos aparecem como sinônimos. Em outros, o território é entendido de maneira genérica e descritiva, referindo-se a uma porção delimitada no espaço. Há, ainda, a perspectiva que trata o território como um tipo específico de espaço, como na clássica definição de Souza (2013), para quem o território é "definido por e a partir das relações de poder", portanto um tipo de espaço recortado por relações de poder.

(3) Para a maioria das categorias, procedeu-se à compatibilização da CBO Domiciliar utilizada pelo IBGE no Censo de 2010 com as Cats utilizadas por Mendonça (2003). Porém, em alguns casos, ocupações deixaram de existir e outras foram incorporadas, o que levou ao redesenho de algumas categorias.

(4) Os dados do CNES foram obtidos indiretamente através da Estação de Pesquisa de Sinais de Mercado do Nescon/UFMG. Os dados da Rais foram obtidos diretamente através de solicitação no Sistema Eletrônico do Serviço de Informação ao Cidadão (e-SIC) do Governo Federal. Os dados do censo demográfico foram coletados diretamente no site do IBGE através de download livre. 
(5) Segundo definição do IBGE (2013), uma área de ponderação é uma unidade geográfica formada pelo agrupamento de setores censitários que tem por objetivo aplicar procedimentos de calibração da representatividade das estimativas amostrais. São definidas respeitando os limites administrati-vos e seguindo critérios quantitativos, de contiguidade e de homogeneidade populacional.

\section{Referências}

AGRESTI, A. e FINLAY, B. (2012). Métodos estatísticos para as Ciências Sociais. Porto Alegre, Penso.

ALMEIDA, M. A. S. e GUANZIROLI, C. E. (2013). Análise exploratória espacial e convergência condicional das taxas de crimes em Minas Gerais nos anos 2000. In: XLI ENCONTRO NACIONAL DE ECONOMIA - ANPEC. Anais... Disponível em: https://www.anpec.org.br/encontro/2013/files_l/i12-1d13c09 485009584e18e968236b99022.pdf. Acesso em: 7 set 2016.

AMARAL, P. V. M.; REIS, J. C. e LUZ, L. S. (2010). Características sociodemográficas e localização em relação a serviços de saúde em Minas Gerais. In: XIV SEMINÁRIO DE ECONOMIA MINEIRA. Diamantina. Anais... Disponível em: http://www.cedeplar.ufmg.br/seminarios/seminario_ diamantina/2010/D10A103.pdf. Acesso em: 7 set 2016.

ANDRADE, M. V. et al. (2012). Mercados e concentração no setor suplementar de planos e seguros de saúde no Brasil. Pesquisa e Planejamento Econômico, v. 42, n. 3, pp. 329-361.

ANSELIN, L. (1995). Local indicators of spatial association - LISA. Geographical Analysis, v. 27, n. 2, pp. 93-115.

BARCELLOS, T. M. e MAMMARELLA, R. (2001). Questões teóricas e metodológicas na pesquisa recente sobre as grandes cidades: notas para reflexão. Ensaios FEE. Porto Alegre, v. 22, n. 2, pp. 248-269.

BOURDIEU, P. (2003). “Efeitos de lugar”. In: BOURDIEU, P. (coord.) A miséria do mundo. Petrópolis, Vozes.

BOUSQUAT, A.; COHN A. e ELIAS P. E. (2006). Implantação do Programa Saúde da Família e exclusão sócioespacial no Município de São Paulo, Brasil. Cadernos de Saúde Pública. Rio de Janeiro, v. 22, n. 9, pp. 1935-1943.

CAMPOS, F. E.; MACHADO, M. H. e GIRARDI, S. N. (2009). A fixação de profissionais de saúde em regiões de necessidades. Divulgação em saúde para debate. Rio de Janeiro, n. 44, pp. 13-24.

GIRARDI, S. N. et al. (2016). Impacto do Programa Mais Médicos na redução da escassez de médicos em Atenção Primária à Saúde. Ciência e Saúde Coletiva. Rio de Janeiro, v. 21, n. 9, pp. 2673-2682.

(2011). "Índice de escassez de médicos no Brasil: estudo exploratório no âmbito da Atenção Primária”. In: PIERANTONI, C. R.; DAL POZ, M. R. e FRANÇA. T. (orgs.). O trabalho em saúde: abordagens quantitativas e qualitativas. Rio de Janeiro, Ce-pesc/IMS/Uerj.

HARVEY, D. (2004). Novo imperialismo. São Paulo, Edições Loyola.

IBGE - INSTITUTO BRASILEIRO DE GEOGRAFIA E ESTATístICA (2013). Metodologia do Censo Demográfico 2010. Série Relatórios Metodológicos, v. 41. Rio de Janeiro. 
LEITE, R. P. (2002). Contra-usos e espaço público: notas sobre a construção social dos lugares na Manguetown. Revista Brasileira de Ciências Sociais, São Paulo, v. 17, n. 49, pp. 115-172.

MARCUSE, P. (2004). Enclaves, sim; guetos, não: a segregação e o Estado. Espaço e Debates. São Paulo, v. 24, n. 45, pp. $24-33$.

MARQUES, E.; SCALON, C. e OLIVEIRA, M. A. (2008). Comparando Estruturas Sociais no Rio de Janeiro e em São Paulo. Dados - Revista de Ciências Sociais. Rio de Janeiro, v. 51, n. 1, pp. 215-238.

MENDONÇA, J. G. (2003). “Belo Horizonte: metrópole segregada”. In: MENDONÇA, J. G. e GODINHO, M. H. L. (orgs.). População, espaço e gestão na metrópole: novas configurações, velhas desigualdades. Belo Horizonte, PUC Minas.

MENDONÇA, J. G. e MARINHO, M. A. C. (2015). “As transformações socioespaciais na Região Metropolitana de Belo Horizonte". In: ANDRADE, L. T.; MENDONÇA, J. G.; INIZ, A. M. A. (eds.). Belo Horizonte: transformações na ordem urbana. Rio de Janeiro, Letra Capital e Observatório das Metrópoles/ Belo Horizonte, PUC-Minas.

MONKEN, M. et al. (2008). “O território na saúde: construindo referências para análises em saúde e ambiente". In: MIRANDA, A. C. et al. (orgs.). Território, ambiente e saúde. Rio de Janeiro, Fiocruz.

MOYA, M. E. (2011). "Os estudos sobre a cidade: quarenta anos de mudança nos olhares sobre a cidade e o social". In: KOWARICK, L. e MARQUES, E. (orgs.). São Paulo: novos percursos e atores - sociedade, cultura e política. São Paulo, Editora 34 e Centro de Estudos da Metrópole.

PAIM, J. et al. (2011). O sistema de saúde brasileiro: história, avanços e desafios. Lancet, Série Saúde no Brasil 1, pp. 11-31.

PONG, R. W. e PITBLADO, J. R. (2002). Beyond counting heads: some methodological issues in measuring geographic physicians. Canadian Journal of Rural Medicine, n. 7, v. 1, pp. 12-20.

PRÉTECEILLE, E. (2004). A construção social da segregação urbana: convergências e divergências. Espaço e Debates. São Paulo, v. 24, n. 45, pp. 11-23.

PRÉTECEILLE, E. e RIBEIRO, L. C. Q. (1999). Tendências da segregação social em metrópoles globais e desiguais: Paris e Rio de Janeiro nos anos 80. Revista Brasileira de Ciências Sociais. São Paulo, v. 14, n. 40, pp. 143-162.

RODRIGUES, C. G.; AMARAL, P. V. M. e SIMÕES, R. F. (2007). Rede urbana da oferta de serviços de saúde: uma análise multivariada macrorregional - Brasil, 2002. Revista de Desenvolvimento Econômico. Salvador, ano IX, n. 16, pp. 83-92.

SOUZA, M. L. D. (2013). Os conceitos fundamentais da pesquisa socioespacial. Rio de Janeiro, Bertrand Brasil.

SOUZA, R. C. F. et al. (2016). Viver próximo à saúde em Belo Horizonte. Cadernos Metrópole. São Paulo, v. 18, n. 36, pp. 325-344.

THERBORN, G. (2006). "Why and how places matters". In: GOODIN, R. e TILLY, C. (eds.). The Oxford Handbook of contextual political analysis. Oxford, OUP.

TRAVASSOS, C. et al. (2000). Desigualdades geográficas e sociais na utilização de serviços de saúde no Brasil. Ciência e Saúde Coletiva. Rio de Janeiro, v. 5, n. 1, pp. 133-149.

TORRES, H. G. e BICHIR, R. (2007). Consequências da segregação residencial para as políticas públicas: o caso do atendimento básico em saúde em São Paulo. Caderno CRH. Salvador, v. 20, n. 50, pp. 245-259. 
TORRES, H. G. (2006). Demografia urbana e políticas sociais. Revista Brasileira de Estudos Populacionais. São Paulo, v. 23, n. 1, pp. 27-42.

VASCONCELOS, C. M. e PASCHE, D. F. (2008). “O Sistema Único de Saúde”. In: CAMPOS, G. W. S. (org.). Tratado de saúde coletiva. São Paulo, Hucitec / Rio de Janeiro, Fiocruz.

Texto recebido em 7/set/2016

Texto aprovado em 7/nov/2016 